\title{
DETERMINATION OF THE VALENCE-BAND OFFSET OF CAS/CIS SOLAR CELL DEVICES BY TARGET FACTOR ANALYSIS
}

\author{
D. W. Niles, M. Contreras, K. Ramanathan, and R. Noufi \\ National Renewable Energy Laboratory \\ 1617 Cole Boulevard, Golden, CO 80401
}

\begin{abstract}
X-ray photoemission spectroscopy (XPS) is used to determine and compare the valence-band offsets $\left(\Delta E_{V}\right)$ for CdS grown by chemical bath deposition on singlecrystal and thin-film CulnSe 2 (CIS). The thin-film CIS device was suitable for photovoltaic energy production. By sputtering through the $\mathrm{CdS} / \mathrm{CIS}$ interface and reducing the depth profile with target factor analysis, the magnitude of $\Delta \mathrm{E}_{\mathrm{V}}$ was determined to be $\Delta \mathrm{E}_{\mathrm{V}}=1.06 \pm 0.15 \mathrm{eV}$ for both the single-crystal and thin-film interfaces. This determination of $\Delta \mathrm{E}_{\mathrm{V}}$ is about $0.25 \mathrm{eV}$ larger than many previously reported estimations CdS grown by physical vapor deposition on CIS and helps explain the record performance of $\mathrm{CdS} / \mathrm{CIS}$ photovoltaic devices.
\end{abstract}

\section{INTRODUCTION}

Solar-cell devices made from the CdS/CulnSe2 (CdS/CIS) heterojunctions are promising candidates for thin-film photovoltaic (PV) energy production [1]. The current record efficiency for CIS-based solar-cell devices exceeds $17 \%$ [2]. Despite progress in the efficiency of CdS/CIS-based solar-cell devices, a complete understanding of the electronic structure of the CdS/CIS interface remains elusive. In this manuscript, we attempt to resolve the controversy involving the magnitude of the valence-band offset $\left(\Delta E_{V}\right)$ and conduction-band offset $\left(\Delta \mathrm{E}_{\mathrm{C}}\right)$ between $\mathrm{CdS}$ and $\mathrm{CIS}$ in thin-film devices [3-6].

The difference in the optical bandgaps of CdS and CIS must be shared by $\Delta E_{V}$ and $\Delta E_{c}$. For efficient $P V$ performance, $\Delta E_{V}$ must be large (approaching $E_{g}[\mathrm{CdS}]-E_{g}[\mathrm{ClS}]=1.38 \mathrm{eV}$ ) and $\Delta E_{c}$ must be small. In fact, early indirect photoemission measurements gave $\Delta \mathrm{E}_{\mathrm{V}}=1.4 \mathrm{eV}$ in support of efficient PV performance [3].

Subsequent direct photoemission measurements yielded $\Delta E_{V}=0.8 \pm 0.1 \mathrm{eV}$ [6]. Our own photoemission measurements have shown the $\Delta E_{V}=0.8 \pm 0.1 \mathrm{eV}$ for CdS on both single-crystal CulnSe 2 and thin-film CIS [7]. The inconsistency between the photoemission-measured $\Delta \mathrm{E}_{\mathrm{V}}$ and the required $\Delta E_{v}$ for effective solar-cell performance has led to other models for the CdS/ClS interface $[4,5]$.

A fundamental difference between the idealized photoemission experiments and the real world of CdS/CIS devices is the method of producing the CdS layer: whereas record-setting $\mathrm{CIS}$ devices employ a CdS layer made by chemical bath deposition (CBD), photoemission measurements have been made on CdS grown in vacuum by physical vapor deposition (PVD). The purpose of these experiments is to bridge the gap between measured valence-band offsets and record device performance by measuring the band alignment for CBD CdS deposited on CIS. To this end, we have deposited CBD CdS onto both single-crystal and thin-film CIS and then performed XPS depth-profiles to determine $\Delta \mathrm{E}_{\mathrm{V}}$.

\section{EXPERIMENT}

CdS layers $50 \mathrm{~nm}$ thick were grown by CBD onto two substrates: one single-crystal CIS (SC-CIS) and one thinfilm CIS (TF-CIS) several microns thick suitable for a highefficiency photovoltaic device. The details of the thin-film CIS growth and CBD processes are described elsewhere $[8,9]$. We then sputtered through the CdS/CIS interface while monitoring core levels and valence bands with $X$-ray photoemission spectroscopy (XPS). The XPS system was a Physical Electronics 5600 XPS equipped with a monochromated $\mathrm{Al} \mathrm{K \alpha}$ source operating at $350 \mathrm{~W}$ and hemispherical analyzer operated at $12 \mathrm{eV}$ pass energy and $800 \mu \mathrm{m}$ spot size.

We used $3 \mathrm{kV} \mathrm{Ar}+$ ions at $\sim 30^{\circ}$ from normal incidence to sputter through the interface while rotating the sample and rastering the ion beam over a $4 \times 4 \mathrm{~mm}$ area. We determined the average sputter rate by measuring the sputter crater depths with a profilometer to be $1.8 \mathrm{~nm} / \mathrm{min}$. Aside from $<1$ at. $\% O$ in the CdS thin-film, we did not observe any residual contaminants from the CBD growth process at the CdS/CIS interface. All atomic concentrations were determined using standard 
sensitivity factors from Ref. 10. All spectra were aligned to the Fermi level of a metaliic In sample.

After obtaining depth profiles from the CdS/SC-CIS and CdS/TF-CIS interfaces, we reduced the depth-profile spectra with target factor analysis (TFA) and determined the $\Delta E_{V}$ from the reconstructed data [11]. TFA is a matrixbased algorithm that is becoming a standard datareduction technique in the analysis of depth profiles. Ref. 11 presents a thorough discussion of TFA.

\section{RESULTS AND DISCUSSION}

Preferential sputtering was not apparent through the CdS overlayer. The atomic concentrations of sputtered thin-film and single crystal CdS were $\mathrm{Cd}=51 \pm 2$ at. $\%$ and $\mathrm{S}=49 \pm 2$ at. $\%$. We did not observe any evidence for metallic $\mathrm{Cd}$ from high-resolution core line spectra or Fermilevel spectra.

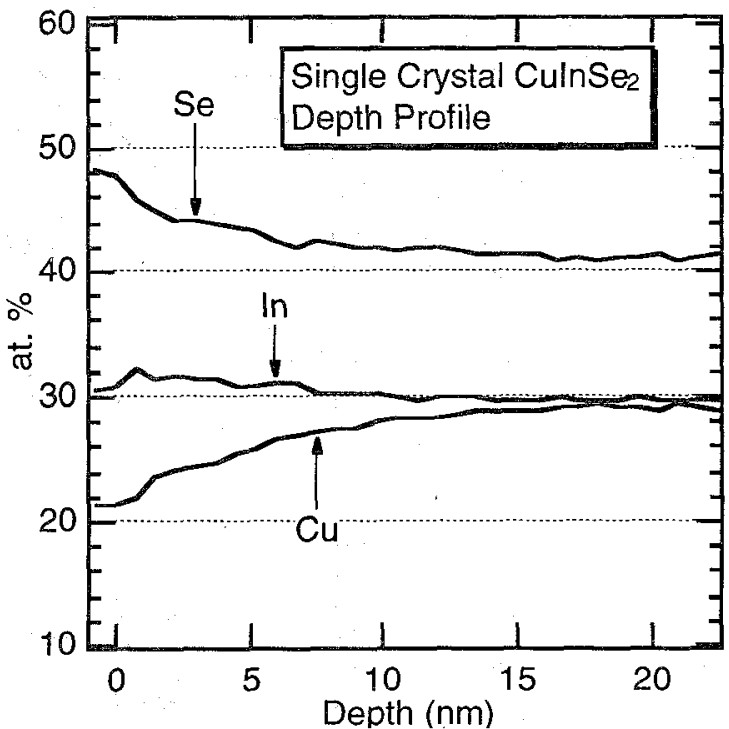

Fig. 1: Atomic concentrations for sputtered single-crystal CIS.

Preferential sputtering was a problem for the CIS substrate. Fig. 1 shows the atomic concentrations of SCCIS cleaved in an $\mathrm{N}_{2}$ ambient, inserted immediately into the analysis chamber, and then sputtered at $3 \mathrm{kV}$. The $O$ signal was $<1$ at. $\%$ and the $\mathrm{C}$ signal was imperceptible. The average atomic concentrations of the bulk were $\mathrm{Cu}=$ 25 at. $\%$, In $=25$ at. $\%$, and $\mathrm{Se}=50$ at. $\%$ as determined by electron microprobe analysis. The crystal appeared to cleave along an imperfection and leave a surface that did not have the same atomic concentrations as the average. Sputtering further decreased the Se content of the CIS surface region and left behind metallic in and $\mathrm{Cu}$.

The metallic behavior of the cations was visible in the In 3d spectrum shown in Fig. 2 and the presence of Fermi level emission (not shown here). Compared to the cleaved crystal, sputtering broadens the In $3 d$ toward lower binding energy and makes a clear metallic in shoulder. The broadening indicates numerous chemical environments for the $\mathrm{In}$, ranging from relatively unperturbed CIS to metallic In.

From nuclear stopping cross-section theory, we estimate a dose of $\sim 10^{15}$ ions $/ \mathrm{cm}^{2}$ and an $\mathrm{Ar}^{+}$ion penetration depth of $20 \mathrm{~nm}$. Therefore, all of the intensity. in the measured photoemission signal stems from the interface region potentially damaged by the ion-beam.

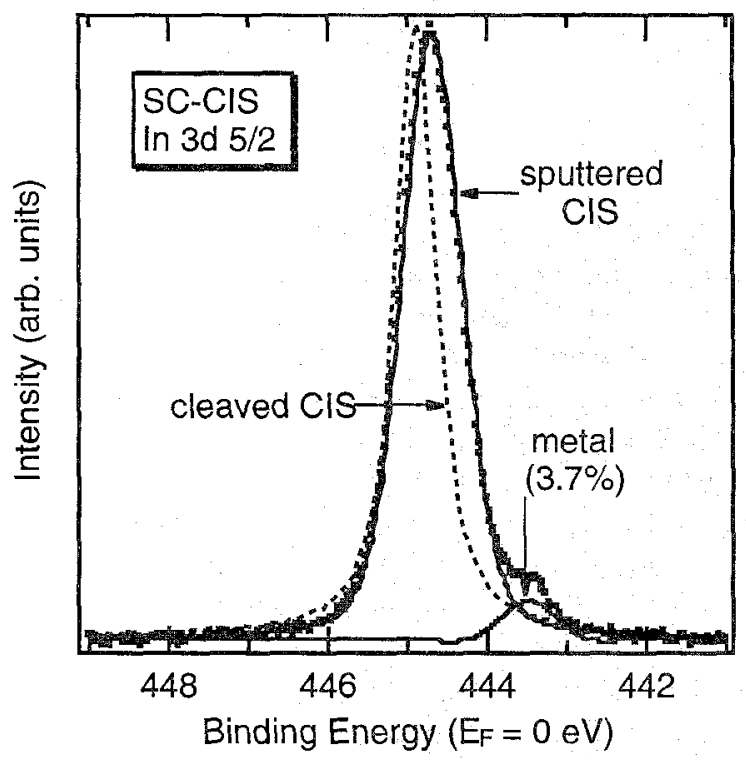

Fig. 2: In $3 d$ 5/2, showing metallic In formation.

Fig. 3 shows the eigenvalue analysis of the depth profiles for both the CdS/SC-CIS and CdS/TF-CIS interface obtained through factor analysis. One can see from the Fig. 3 two primary eigenvalues followed by smaller ones that are about 3 orders of magnitude smaller that the two primary ones. Analysis of the residual error, the IND function, and eigenvectors associated with the eigenvalues support the conclusion that only two factors are needed to describe the depth profiles [11].

Fig. 4 shows these two factors for the CdS/SC-CIS interface after target rotation: obviously, they correspond to the CdS overlayer spectrum and the CIS substrate spectrum. The conclusion is that every spectrum of the depth profile is a linear superposition of the spectrum of the CdS overlayer and the ion-damaged CIS substrate.

In Fig. 4, one can see embedded $\mathrm{Cd}$ in the CIS substrate, metallic In, and Fermi-level emission (not visible in the spectra as plotted) from the metallic cations. The amount of $\mathrm{Cd}$ in the CIS film is difficult to determine because the intensity is weak, but is less than 1 at. \%. To within experimental error, the valence-band emission after sputtering with $\mathrm{Ar}^{+}$was not significantly different from the 
the SC-CIS factor shown in Fig. 4. The fact that we need only two factors to describe the depth profile despite the problems brought about by preferential sputtering is somewhat perplexing. One would expect a priori to need three factors: one for the CdS, one for the CIS before sputtering created significant damage, and one for the heavily damaged CIS at the end of the depth profile. Our conclusion is that the shape of the CIS valence-band emission is relatively insensitive to the level of sputterinduced damage.

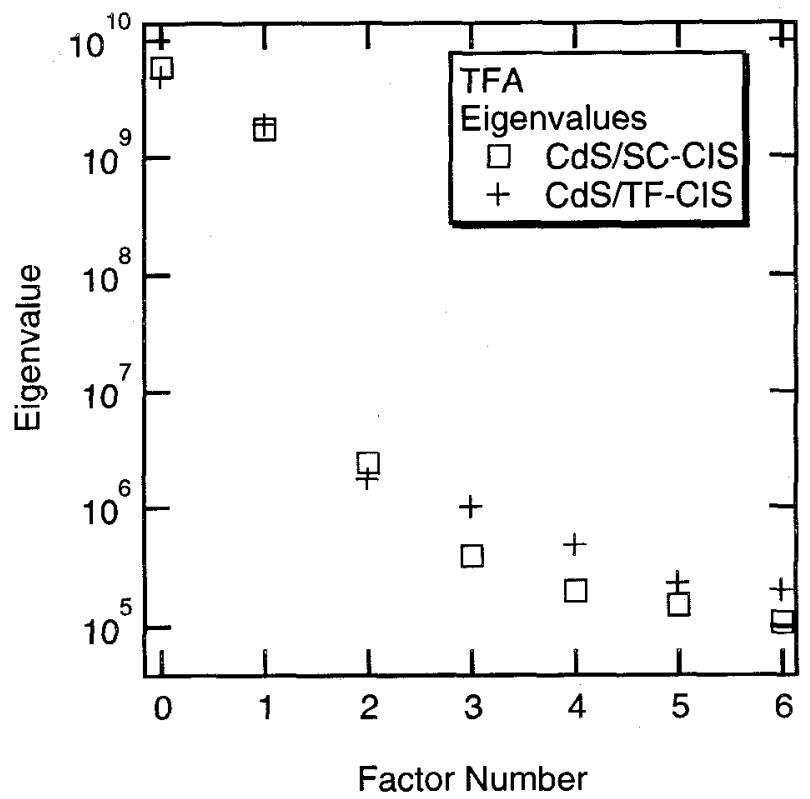

Fig. 3: Eigenvalues for both interfaces, showing that only two factors are necessary reconstruct the depth profile.

Further support for this conclusion comes from reducing the valence-band emission of the depth profile on the cleaved single-crystal (atomic concentrations shown in Fig. 1). We first measured a spectrum from a metallic in sample, and used it to subtract the metallic In signal from the cleaved, sputtered SC-CIS spectra. We then reduced the remaining emission with TFA, and found that one factor accounted for the signal, to within experimental error.

We used reconstructed data from the factors in Fig. 4 and the loading factors to determine $\Delta E_{V}$ for the system as a function of sputter depth. To determine the VBM, we linearly extrapolated the leading edge of the valence-band signal. We also determined the accuracy by taking the largest and smallest acceptable extrapolations consistent with the data. Fig. 4 is a plot of $\Delta \mathrm{E}_{\mathrm{V}}$ and the loading factors.

The data points and the smooth curve in Fig. 5 represent the position of the valence-band maximum (VBM) as a function of sputter depth. The dashed curves in Fig. 5 are the CdS and CIS loading factors as a function of depth. At depths less that $40 \mathrm{~nm}$, the photoemission signal looks like the CdS factor seen in Fig. 4, without any signal from the CIS present. Between 40 and $60 \mathrm{~nm}$, the depth-profile spectra change from appearing like CdS to appearing like CIS (with some embedded $\mathrm{Cd}$ ). Within this transition region the position of the VBM moves towards the Fermi level. $\Delta \mathrm{E}_{\mathrm{V}}=1.01 \mathrm{eV}$ is the total change in the position of the VBM.

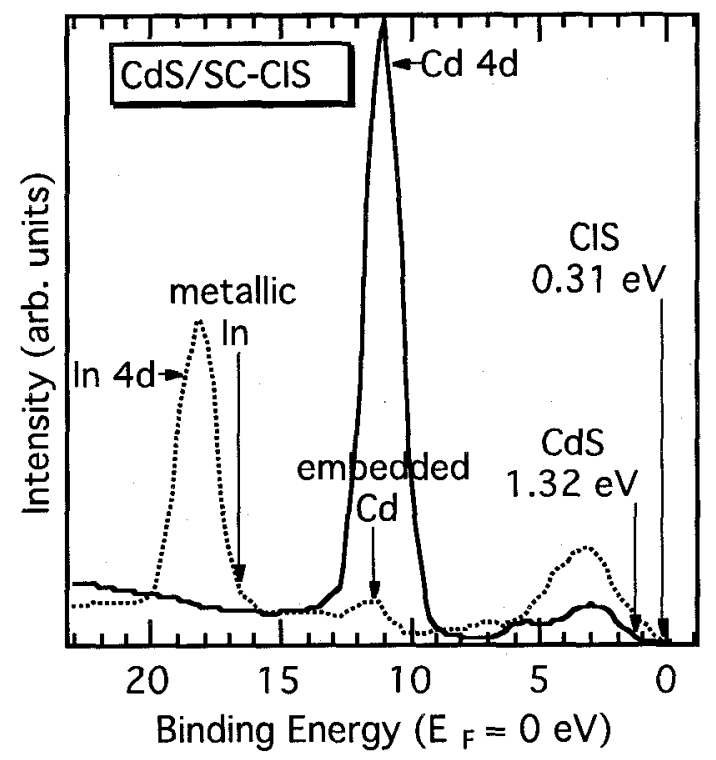

Fig. 4: CdS and CIS factors for the CdS/SC-CIS interface.

The results from the CdS/TF-CIS interface are essentially identical in all respects, except we measure $\Delta E_{V}=1.11 \mathrm{eV}$. Fig. 6 shows the results. To within experimental error, this measured $\Delta E_{V}$ is that same as for the $\mathrm{SC}$-CIS substrate. We conclude that $\triangle \mathrm{E}_{\mathrm{V}}$ for CBD CdS on both thin-film and single-crystal CIS is $\Delta E_{V}=1.06 \pm$ $0.15 \mathrm{eV}$.

In an earlier set of experiments, we deposited CdS by PVD at room temperature on both SC-CIS and TF-CIS while monitoring the valence-band emission with synchrotron radiation photoemission. For these systems, we found $\Delta \mathrm{E}_{\mathrm{V}}=0.8 \pm 0.1 \mathrm{eV}$ in agreement with other measurements $[6,7]$. Although the error bars on the CBD and PVD results prohibit absolute confirmation of a larger $\Delta E_{V}$ for $C B D$ devices, the data for the CBD CaS were reproducible and consistently showed a larger $\Delta \mathrm{E}_{\mathrm{V}}$.

A possible explanation for the difference is the presence of interface contaminants. Except for a small $O$ signal ( $\leq 1$ at. $\%$ ), we did not see any contamination. Another possible explanation is that CBD and PVD CdS are chemically different. However, we carefully analyzed the atomic compositions, high-resolution photolines, and Auger parameters of $C B D, P V D$, and single-crystal CdS without uncovering any fundamental differences.Our present thesis for the difference in $\Delta E_{V}$ is that the 
CdS/CIS interface chemistry is different between CBD and PVD CdS, but ion-beam damage obscures the chemistry of the interface and renders our data insufficient to prove this thesis.

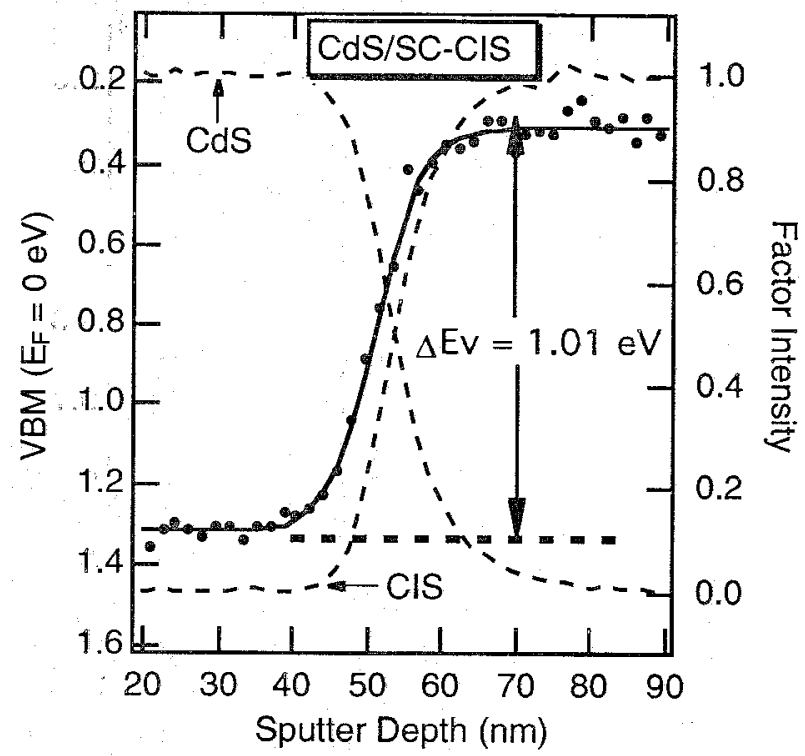

Fig: 5 : Loading factors and $\Delta E_{V}$ for the CdS/SC-CIS interface.

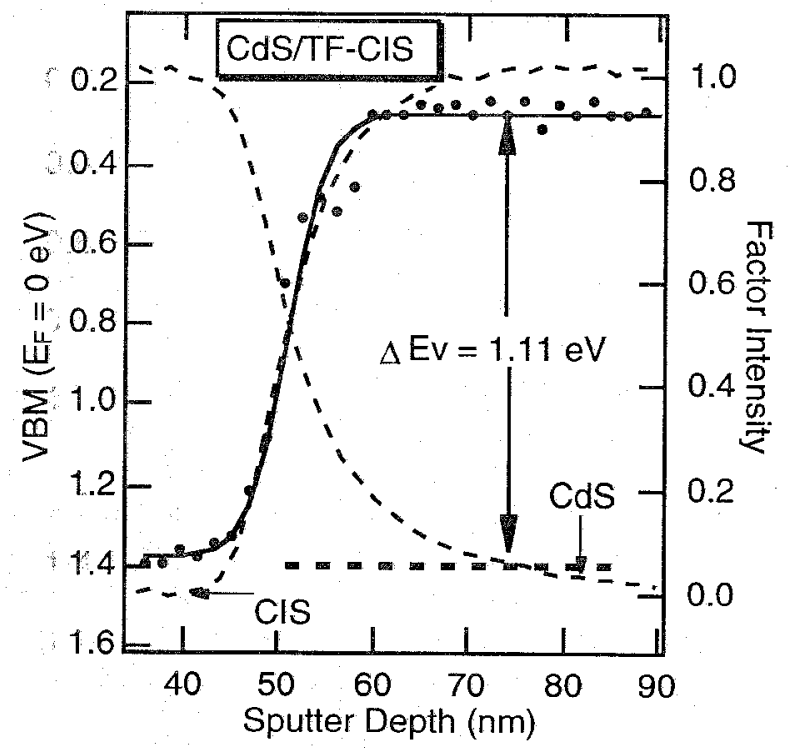

Fig. 6 Loading factors and $\Delta E_{V}$ for the CdS $/ \mathrm{TF}-\mathrm{CIS}$

The important quantity with regard to the performance of photovoltaic devices is the conduction band offset $\left(\Delta E_{c}\right.$ ) rather than the valence band offset. The general assumption within the photovoltaic community is that $\Delta \mathrm{E}_{\mathrm{C}}$ $<0.3 \mathrm{eV}$ is necessary to understand the flow of charge across the CdS/CIS interface [12]. Using our measured $\Delta E_{V}, E g(C d S)=2.42 \mathrm{eV}$ and $E g(C I S)=1.04 \mathrm{eV}$, we calculate $\Delta E_{C}=0.32 \pm 0.15 \mathrm{eV}$. Our calculated value of $\Delta E_{C}$ for a real device supports the traditional model for CdS/CIS-based solar cells.

\section{REFERENCES}

[1] H. Ullal et al., Twenty-fourth IEEE PVSC, 1994, v. 1, p. 266.

[2] M. A. Green et al., Prog. Photovoltaics 3, 229 (1995).

[3] M. Turowski et al., Phys. Rev. B 31, 1022 (1985).

[4] D. Schmid et al., J. Appl. Phys. 73, 2902 (1993).

[5] S.-H. Wei et al., Appl. Phys. Lett. 63, 2549 (1993).

[6] T. Löher et al., J. Appl. Phys. 77, 731 (1995).

[7] D. W. Niles, unpublished.

[8] M. Contreras et al., Twenty-fourth IEEE PVSC, 1994, v. 1, p. 68

[9] J. Kessler et al., Sixth International PVSEC 1992, p. 1005.

[10] Jill Chastain, ed., Handbook of X-Ray Photoelectron. Spectroscopy, edited by (Physical Electronics, Eden Prairie, MN,1992).

[11] E. R. Malinowski, Factor Analysis in Chemistry by (Wiley and Sons, Inc., 1991).

[12] J. L. Gray et al., AIP Conference Proceedings 353, 1995 p. 345. 